\title{
Write a departmental handbook for junior staff
}

\author{
Janet McDonagh, Fiona Clarke, Dan Veale
}

Many medical senior house officer rotations now change every four months. Thus senior house officers arrive on a new unit expected to manage complicated cases, handle specialist clinics, and explain details to patients when they have previously seen such problems only briefly as students. They generally rely on help and answers to their questions from middle grade staff and nurses. The pace of the health service does not allow time to cancel clinics and admissions for induction days, even for the best intentioned reasons, and frequently other staff are too busy to sort all problems. Biannual induction courses usually have a general remit-for example, revision of basic skills and information about administrative arrangements and local practice-and do not answer the more specific queries for each of the many subspecialties. In addition, in a recent survey of preregistration house officers $36 \%$ could not attend such a course. ${ }^{1}$ Thus junior doctors find great comfort in having a written manual available which is directed towards the unit and specialty concerned.

\section{Proposed handbook}

Such a handbook also facilitates quick integration into the department. Ideally, it should be sent to the junior doctor before he or she starts the post and the doctor should be allowed to keep it when he or she leaves. A copy should always be available in the junior medical staff office on the ward. A plethora of copies around the unit and in clinic rooms would make it readily accessible. We appreciate the smallness of the junior staff white coat pocket, and the handbook we propose is an informative reference book rather than just another emergency medicine handbook. The handbook can also be a stimulus for further learning and should be written in an educational rather than didactic manner. It could also incorporate such features as a log diary and a programme of educational objectives for that particular post, which would be regularly reviewed with a named educational supervisor. ${ }^{2}$ Junior doctor posts are meant to be educational, and a departmental handbook shows that senior staff are aware of the practical problems that arise for juniors and that they are prepared to help.

This article is based on our experiences of compiling a handbook for junior medical staff in the departments of rheumatology at the Freeman Hospital and the Royal Victoria Infirmary, Newcastle upon Tyne.

Newcastle upon Tyne NE1 4LP

Janet $\mathrm{McD}$ Donagh, research registrar, department of rheumatology

Dan Veale, senior registrar, department of medicine

Freeman Hospital, Newcastle upon Tyne NE7 7DN

Fiona Clarke, registrar, department of rheumatology

Correspondence to: Dr McDonagh.

$B M^{7} 1993 ; 307: 553-5$

\section{Whose job is it?}

The handbook should be compiled by a named junior member of staff in post-for example, a registrar with experience in the specialty-with the support and advice of a sympathetic consultant. This ensures that only important, useful, and relevant information is included. Updating protocols and thinking about "how to do it" issues provide important educational experience for a registrar in higher professional training. Word processing facilities should ensure that such handbooks do not become out of date, each generation of staff modifying the booklet so that it remains current.

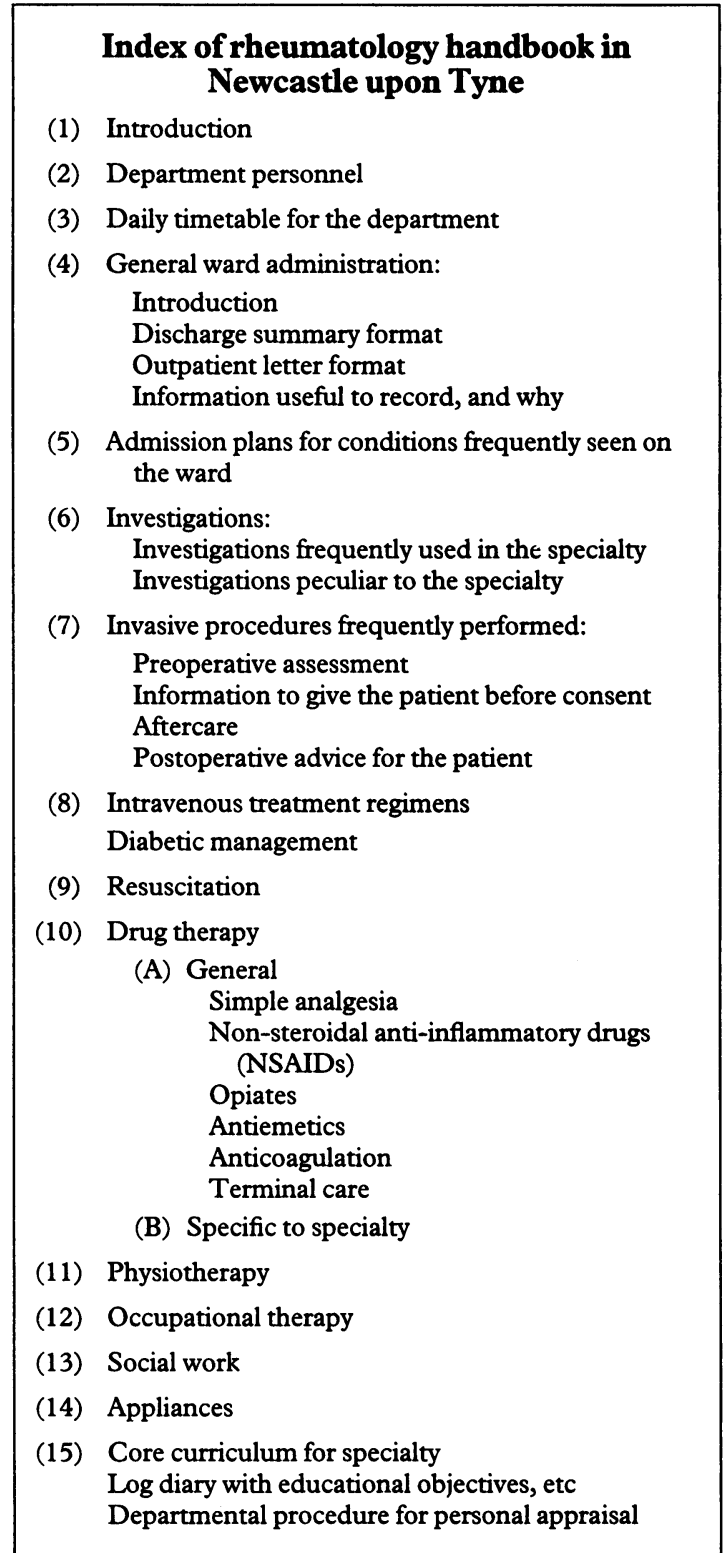

\section{Where to start?}

Requests for ideas and suggestions for what should be included in such a handbook should be made to all members of the department. Outcomes of departmental audit may provide relevant guidelines or protocols, or both, as well as identifying key aspects of clinical practice that warrant explanation. For some conditions national guidelines are now available, which could be outlined or referenced. The handbook in our rheumatology departments began as a booklet for our drug monitoring clinics, which had been subjected to audit.

\section{What to include}

The contents of the handbook may be considered in two sections-general information and information specific to the specialty (see box). 
GENERAL INFORMATION

\section{Department personnel and their timetables}

This section is essentially a "Who's Who" of staff within the department-that is, secretaries, senior and specialist nursing staff, specialist paramedical staff, and senior medical staff-and includes details of their locations. Names of the consultants responsible for supervising junior staff, the name of the postgraduate tutor, as well as the names of key contact people in related departments such as laboratories and university departments may also be useful, as is a list of telephone numbers for all these people.

\section{Departmental routine}

A timetable of ward rounds and clinics should be detailed as well as the relevant postgraduate timetable of departmental clinical, audit, pathology, and radiology meetings. With the development of appraisal for trainees in hospital medicine, as recommended by the Royal College of Physicians, any such mechanism within the department could be outlined in the handbook. Ideally, a core curriculum for senior house officer-preregistration house officer training for the particular specialty could also be included, with a checklist or log diary, or both, to monitor adequate coverage of topics in teaching sessions as well as clinical experience.

\section{Administration}

Skeleton formats for outpatient letters and discharge summaries should be provided. We have included explanations of the computer programs for these in our department. The importance of accurate note keeping should be emphasised and reference made to any particular expectations of documentation peculiar to the specialty or department. We recommend that the Royal College of Physicians' form for case note audit should be included.

\section{Communication}

Communication with general practitioners and other colleagues should be encouraged and warrants specific mention, especially with respect to the following: at time of discharge of certain patients, such as those with a newly diagnosed malignancy; after the introduction of a new drug that will need monitoring; after a patient dies.

\section{General patient care}

On many specialty wards such aspects of patient management as analgesia, antiemesis, diabetes, intravenous treatment regimens, and terminal care are often left to junior staff who may not be experienced in such matters. In a recent survey almost two thirds of preregistration house officers had never been adequately taught how to manage pain. ${ }^{1}$ Including relevant information on these topics may therefore be useful for all departmental handbooks.

Analgesia-Rather than giving a long list of painkillers, consider including the following in an attempt to encourage a more rational and educated analgesic regimen: (a) a summary of the types of pain (visceral, bone, nerve), with reference to means of relieving pain in these different circumstances; (b) the analgesic "staircase" concept, starting with the non-opioids and moving towards the stronger opioids; $(c)$ a conversion table for the different formulations of opioids (not easily accessible in the British National Formulary, yet frequently required by resident junior staff); (d) details of the use of coanalgesics such as corticosteroids and antispasmodics; ${ }^{3}$ (e) reference to non-drug analgesiafor example, transcutaneous electrical nerve stimulation.

Antiemetics-As with analgesia the inclusion of a summary of the possible mechanisms of nausea and vomiting encourages more rational prescribing not always facilitated by the six pages of drugs in the British National Formulary, not all of which may be available in every hospital. (Local formularies, when they exist, are helpful in this regard.)

Anticoagulation-The locally approved regimen for anticoagulation is useful as well as the therapeutic ranges for the various underlying conditions.

Intravenous treatment-The constitution of commonly used intravenous fluids should be detailed together with guidelines for their use in uncomplicated cases, elderly patients, renal or hepatic impairment, and the immediate postoperative period. This may also help encourage rational prescribing for patients. ${ }^{4}$

Diabetes-The care of diabetic patients on nonmedical wards may in some hospitals be supervised by a specific diabetic care team. Where such a service is not available guidelines for diabetic management may usefully be included, especially with reference to preoperative and postoperative management. ${ }^{5}$

Resuscitation-The current national guidelines could be included ${ }^{6}$ and reference made to any local courses that may be available for practical appraisal of resuscitation skills.

Terminal care is often neglected on busy hospital wards and, as mentioned above, analgesia, antiemesis, and relief of constipation and so on are often left to the most junior member of the medical staff, who may not have the necessary clinical experience. We recommend reference to $A$ Guide to Symptom Relief in Advanced Cancer. ${ }^{3}$

INFORMATION SPECIFIC TO THE SPECIALTY Assessment plans

In any specialty there will often be a limited range of routine admissions. The provision of assessment plans for such patients compiled with an educational rather than a didactic emphasis is preferred and is particularly welcomed by the junior staff in our department. Care should be taken to explain why recording certain information is important, so that patients with a chronic disease like rheumatoid arthritis are no longer treated as routine admissions or subjects for quick clerking. As keeping case notes is the job of junior staff, giving such information is crucial if we wish to remain active in clinical research and audit, which so often entail reviewing case notes.

\section{Procedures}

Specialties often have their own selection of procedures regularly performed on the ward, and details of these should be included under the headings of preparation; explanation for the patient of what to expect, including any risks; technique; and aftercare, including signs and symptoms of any complications. We recommend Procedures in Practice as a book to have handy in any junior medical staff room on a ward. ${ }^{7}$

\section{Investigations}

Specialisation in medicine is accompanied by an ever lengthening list of investigations. On some wards long lists of investigative protocols adorn the walls of the junior doctors' offices. Such lists may need to be included in the handbook, but when possible information on the indications for and relevance of such tests should also be included.

\section{Drugs specific to the specialty}

Details of any drugs primarily used in the specialty should be included with information about any necessary advice for patients, adverse reactions, monitoring regimens, and drug interactions. Protocols for chemotherapy regimens regularly given on the ward should be carefully detailed. 


\section{Related disciplines}

Most medical specialties have links with other medical and paramedical disciplines. Details of the services provided by such disciplines can be mentioned in the handbook. We find that such information is usefully gathered by visiting the departments, which can be included in any induction course. The handbook should contain details of the referral information required by the particular professionals concerned. When describing social work and appliances it might be useful to reference Understanding Benefit ${ }^{8}$ and More Everyday Aids and Appliances," which should also be available on the ward when possible.

\section{Conclusions}

These are only guidelines and every specialty will have its own requirements. We also emphasise that a departmental handbook should be regarded not as an alternative to in service training but as a support and hopefully a stimulus for further learning. Ideally, it should be distributed to junior doctors before the relevant module and should be available for them to take away when they leave for their next job. We must no longer depend on "osmotic learning" as the sole basis of postgraduate medical education, and we must protect, encourage, and stimulate the enthusiasm of young doctors to continue learning in the everyday life of busy hospital wards.

We thank the junior staff for constructive criticism of the first edition of our handbook; Dr Paul Crook for interest, advice, and support; and the Newcastle Rheumatic Research and Education Fund, which financed the project.

1 Dent THS, Gillard JH, Aarons EJ, Crimlisk HI, Smyth-Pigott PJ. Preregistration house officers in the four Thames regions: I. Survey of education and workload. BMF 1990;300:713-6.

2 Baker M. Enhancing the educational content of SHO posts. BMF 1993;306: 808-9.

3 Regnard CFB, Davies A. A guide to symptom relief in advanced cancer. Manchester: Haigh and Hochland, 1986.

4 Dunn DC, Rawlinson N. Surgical diagnosis and management-a guide to general surgical care. London: Blackwell, 1988:83-92.

5 Gill GV, Alberti KGGM. Surgery and diabetes. Hospital Update 1989;15: 327-36.

6 Chamberlain DA. Advanced life support. BMF 1989;299:446-8.

7 Procedures in practice. London: BMJ Publishing Group, 1987.

8 Ennals S. Understanding benefits. London: BMJ Publishing Group, 1991.

9 Mulley G, ed. More everyday aids and appliances. London: BMJ Publishing Group, 1991.

(Accepted 2 Fuly 1993)

\section{Letter from Brasilia}

\section{Chronic malaria syndromes}

Philip Marsden

Chronic malaria is not a popular concept, especially among older doctors, because of the number of ex-servicemen who claimed compensation for it after the second world war. One of the most convincing temperature charts of 48 hour cyclical fever I have seen was that of a man who had not left London for 10 years. He had his first bout of "malaria" while cleaning the Albert Memorial. The eventual diagnosis was colonic carcinoma with secondary pyogenic emboli. As in this man's experience, once you have known malaria's classic sequence of chills, high fever, and drenching sweats it is natural to think of its return, especially since everybody knows that it is a relapsing infection. Of the four types of human malaria, Plasmodium falciparum can relapse for up to two years in an immune subject where initial treatment has been inadequate, and $P$ vivax and $P$ ovale for up to five. $P$ malariae persists latent in the circulation for decades, which is why it figures prominently in the literature of transfusion malaria.

I remember analysing case notes of patients admitted to the Hospital for Tropical Diseases, London, in the early 1960 s with an initial diagnosis of malaria. The two commonest causes were dental root sepsis and urinary tract infection. Today, with reliable serological tests, the possibility of occult malaria can be easily excluded.

I have discussed acute malaria in an earlier letter.' I wish briefly to discuss here the chronic malaria syndromes found in areas of hyperendemic or holoendemic malaria transmission. I always thought, in my years in sub-Saharan Africa, that these syndromes posed ?. great clinical problem for the overworked physician. Here, even in Amazonian Brazil, malaria transmission is usually hypoendemic or mesoendemic. Only when you penetrate up small rivers do you encounter higher rates of transmission. I remember sitting with a colleague on the bank of such a river. $\mathrm{He}$ was wearing only a pair of shorts, and his legs, arms, and trunk appeared spiny because of the female

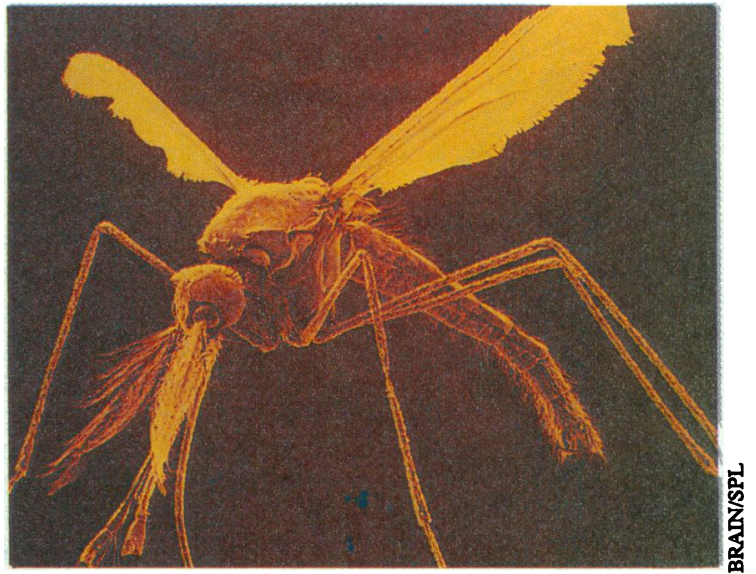

Anopheles gambiae invaded Brazil from Africa before the second world war; had it not been eradicated, would Brasilia have been built?

anopheline mosquitos standing on their heads to suck his blood. He wanted to take a strain of vivax back and would leave Brazil in time to get his first fever in London.

That situation resembles west Africa and the Gambia, where I spent my first long assignment at the Medical Research Council laboratories doing one of the initial studies of African child health. The Gambia is really a vast swamp. When I entered my house, usually after sunset, a hundred Anopheles gambiae mosquitos came in with me through the double screened doors. I dined with them and had to clear my mosquito net before sleep was possible. I took regular effective chemoprophylaxis and never had malaria, although later I got vivax in the New Guinea interior.

In the Gambia, where you are bitten by an infected anopheline every day, I saw how malaria influenced the growth and development of children. They were persistently anaemic, and my laboratory, examining 20-30 slides a day, constantly recorded falciparum 